Conclusions: Activation of ER $\alpha$ significantly enhances the miR processing, and affects the profile of miR transcription in female RA patients. The change in miR profile during E2-treatment could contribute to a significantly change in the miR landscape and disposition of intracellular processes in RA.

Disclosure of Interest: None declared

DOI: 10.1136/annrheumdis-2018-eular.6662

\section{THU0016 TRANSMITOCHONDRIAL CYBRIDS SHOW THAT OXPHOS VIA, BUT NO GLYCOLYSIS VIA, IS INVOLVED IN THE ATP REDUCTION OF OA HUMAN CHONDROCYTES}

M. Fernández-Moreno ${ }^{1,2}$, A. Dalmao-Fernandez ${ }^{2}$, T. Hermida-Gomez ${ }^{2}$, A. Rustan $^{3}$ J. Lund ${ }^{3}$, M.E. Vazquez-Mosquera ${ }^{2}$, S. Relano-Fernandez ${ }^{2}$, I. Rego-Perez ${ }^{2}$, F. Blanco-Garcia ${ }^{2} .{ }^{1}$ CIBER-BBM, Madrid; ${ }^{2}$ INIBIC, A Coruña, Spain; ${ }^{3}$ Department of Pharmaceutical Biosciences, Oslo University, Oslo, Norway

Background: Mitochondrial dysfunction is well documented in OA and has the capacity to alter chondrocyte function and viability, contributing to cartilage degeneration. It is important to evaluate the influence of mitochondria in the pathogenesis of OA using an in vitro model to explain the functional consequences of this association and help us to identify potential diagnostic biomarkers and/or therapeutic targets Transmitochondrial cybrids are a useful cellular model to study the mitochondrial biology and function implications in the cellular behaviour, since they carry different mitochondrial variants with the same nuclear background, therefore, excluding the variations because of nuclear genome.

Objectives: The aim of this work is test mitochondrial activity in the OA chondrocytes using transmitochondrial cybrids with mtDNA from healthy donors (without $\mathrm{OA})$ and from patients with $\mathrm{OA}$.

Methods: Cybrids were developed using 143B. TK Rho-0 cell line (nuclear donor) and platelets (mitochondrial donors) from healthy and OA donors. Human articular chondrocytes were obtained from patients with hip replacement. The mtDNA copy number was measured by real-time PCR method. The ROS production was evaluated using flow cytometry. The metabolic status was evaluated by glucose consumption and glucose oxidation. The glycolytic activity was measure after addition of glucose, oligomycin and 2-dioxyglucose using Seahorse XFp (ECAR). The OXPHOS function was evaluated by SeaHorse XFp (OCR) after addition of oligomycin, FCCP and Rotenone/Antimycin. Appropriate statistical analyses were performed with GraphPad Prism v6.

Results: The analysis of mtDNA copy number showed that the OA have higher levels than $\mathrm{N}$ in cybrids and human chondrocytes. The analysis of ROS production showed that OA had higher levels than $\mathrm{N}$ in both types of cells. The metabolic status analysing glucose consumption, glucose oxidation and total glucose cellular uptake reflected higher values in OA cybrids than $\mathrm{N}$ cybrids. But the analysis of glycolysis data showed lower values in OA than $\mathrm{N}$ cybrids. The analysis of ATP obtained through glycolysis did not show any difference between cybrids. The analysis of OXPHOS function showed that OA had lower basal respiration and maximal respiratory capacity than $\mathrm{N}$ in both types of cells. The ATP obtained via OXPHOS was lower in OA than in $\mathrm{N}$.

Conclusions: The analysis of OXPHOS function supports the participation of mitochondria in cybrids and human chondrocytes metabolism. Both types of cells use the mitochondria to obtain ATP and OXPHOS via, but no glycolysis, is involved in the reduction of ATP synthesis by OA cells. All these data support that $\mathrm{N}$ cybrids and chondrocytes use mitochondria with more efficiency.

Disclosure of Interest: None declared

DOI: 10.1136/annrheumdis-2018-eular.3625

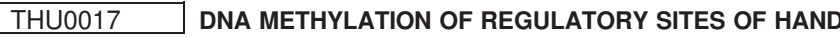 OSTEOARTHRITIS SUSCEPTIBILITY GENES IN FINNISH WOMEN}

S. Hämäläinen, S. Solovieva, T. Vehmas, A. Hirvonen, P. Leino-Arjas. Finnish Institute of Occupational Health, Helsinki, Finland

Background: Despite the hard effort in OA genetic studies only a small part of the estimated effect has been found so far and thus the focus has been changing from genetic to epigenetic studies. The most widely studied epigenetic control mechanism is DNA methylation. There are only few studies on hand OA concerning DNA methylation but their results are promising.

Objectives: Our aim was to study methylation of the regulatory sites of our previously found hand OA susceptibility genes and to replicate some previously reported methylation sites associated with hand OA, in our Finnish women hand OA material.

Methods: The study design was cross-sectional. Bilateral hand radiographs of 542 occupationally active Finnish female dentists and teachers aged 45 to 63 and classified them according to the presence of OA by using reference images. Radiographic hand OA at least mild, in at least three joints (ROA2 3), and radiographic findings summary score (OASUM) were used to define hand OA status. The methylation percentages were determined by bi-sulfite converted DNA pyrosequencing with commercial CpG Assays. Statistical analyses were performed using hierarchical multiple linear regression.

Results: Of the studied methylation sites the COL2A1_1 methylation site was associated with ROA2 $3(\mathrm{p}=0.04)$. Also ALDH1A2 01 methylation site was asso ciated with OASUM $(p=0.02)$. When the data was stratified by occupation the association was only significant, and stronger, in teachers but not in dentists (COL2A1 $1 \mathrm{p}=0.02$ vs. $\mathrm{p}=0.36$ for ROA2 3 , and $A L D H 1 A 2$. $\mathrm{p}=0.01$ vs. $\mathrm{p}=0.36$ for OASUM, respectively). The studied methylation sites in TGFB1, RRP9 and TRAPP5 genes had methylation percentages under the detection limit and they were excluded from the analysis.

Conclusions: Our results lend further support to COL2A1 and $A L D H 1 A 2$ being hand $O A$ susceptibility genes at the epigenetic level.

Acknowledgements: The study was financially supported by a grant from the Finnish Work Environment Fund (113369).

Disclosure of Interest: None declared

DOI: 10.1136/annrheumdis-2018-eular.1067

\section{THU0018 1 MIRNAS-146A AND -499 GENE EXPRESSION AND THEIR POLYMORPHISMS AS DIAGNOSTIC MARKERS} FOR RHEUMATOID ARTHRITIS

G.A. Ayeldeen ${ }^{1}$, Y.H. Nassar ${ }^{1}$, H.A. Ahmed ${ }^{1}$, O.G. Shaker ${ }^{1}$, T.A. Gheita ${ }^{2} .{ }^{1}$ Medical Biochemistry and Molecular Biology; ${ }^{2}$ Rheumatology and Clinical Immunology, Faculty of Medicine, Kasr Al-Ainy School of Medicine, Cairo University, Cairo, Egypt

Objectives: To investigate the expression of miRNAs-146a and -499 and thei polymorphisms in Egyptian patients with RA and to evaluate their relationship to clinical manifestations and disease activity.

Methods: Fifty-two RA patients and 56 matched controls were studied. Disease activity score-28 was assessed. MicroRNAs expression and polymorphisms were assayed by polymerase chain reaction (PCR).

Results: Patients mean age was $39.5 \pm 10.8$ years and the disease duration of 7 \pm 5.1 years. The DAS28 was $3.1 \pm 1.7 ; 23$ were in remission, 5 had mild disease activity, 18 moderate and 6 severe. There was a $15.5 \pm 27.2$ fold increase in miRNA-146a and 3.3 \pm 6.1 in miR-499. The fold change of miRNA-146a was significantly higher in those without joint deformities $(n=18)(28.1 \pm 42.6)$ compared to those with $(8.8 \pm 8.4 ; p=0.01)$. Both miRNA-146a and -499 fold change were significantly decreased in those with a positive ANA $(n=7)(2.5 \pm 2.1$ and $0.07 \pm 0.08) \mathrm{com}$ pared to those with a negative test $(18.1 \pm 29.6$ and $3.8 \pm 6.5 ; \mathrm{p}=0.002$ and $p=0.001$ respectively). The fold changes tended to be higher in those in remission compared to active patients. However, miRNA-499 in those with severe disease activity tended to be higher. There was no significant correlation of the fold change of the miRNAs with the clinical manifestations or medications received. Only ANA positivity significantly inversely correlated with the fold increase in mi-RNA-146a $(r=-0.42, p=0.003)$. The fold change in miRNA-146a significantly correlated with miRNA-499( $r=0.56, p<0.0001)$

Conclusions: Both miRNAs-146a and-499 are highly expressed in RA patients and can be considered as diagnostic markers. Increased expression of miRNA $146 \mathrm{a}$ expression is protective in those with negative ANA and both in those without joint deformities.

Disclosure of Interest: None declared

DOI: 10.1136/annrheumdis-2018-eular.5210

\section{HU0019 \\ ARE MICRORNAS A MOLECULAR CLOCK? THE NEWCASTLE THOUSAND FAMILIES COHORT STUDY}

T.L. Jones $^{1}$, D.A. Young ${ }^{2}$, M.S. Pearce ${ }^{3}$, A. Skelton ${ }^{4}$, F.N. Birrell ${ }^{1}{ }^{1}$ Institute of Cellular Medicine; ${ }^{2}$ Institute of Genetic Medicine; ${ }^{3}$ Institute of Health and Society; ${ }^{4}$ Bioinformatics Support Unit, Newcastle University, Newcastle-upon-Tyne, UK

Background: No single biomarker has been identified for monitoring ageing trajectory. To date, biological clocks are based on DNA methylation, telomere length, p16 $6^{\text {ink4a }}$ expression and microsatellite mutations ${ }^{1}$. Body ageing is a complex phenomenon, including a progressive, pro-inflammatory state, termed 'inflammaging'. MicroRNAs have been linked with cellular senescence and inflammaging. MicroRNAs are short, non-coding sequences of RNA regulating post-transcriptional gene expression with impressive stability and ubiquitous presence, making them ideal candidate biomarkers. Previous studies of circulating microRNAs in ageing were small-scale ${ }^{2}$ or compared individuals of different ages, which is methodologically less robust ${ }^{3}$. MicroRNA biomarkers can bring greater understanding to 